\title{
Epidermal growth factor receptor expression in neurofibromatosis type 1-related tumors and NF1 animal models
}

\author{
Jeffrey E. DeClue, ${ }^{1}$ Sue Heffelfinger, ${ }^{2}$ Giovanna Benvenuto, ${ }^{1}$ Bo Ling, ${ }^{3}$ Shaowei Li, ${ }^{1}$ \\ Wen Rui, ${ }^{3}$ William C. Vass, ${ }^{1}$ David Viskochil, ${ }^{4}$ and Nancy Ratner ${ }^{3}$ \\ ${ }^{1}$ Laboratory of Cellular Oncology, National Cancer Institute, Bethesda, Maryland, USA \\ ${ }^{2}$ Department of Pathology, and \\ ${ }^{3}$ Department of Cell Biology, Neurobiology, and Anatomy, University of Cincinnati College of Medicine, \\ Cincinnati, Ohio, USA \\ ${ }^{4}$ Department of Genetics, University of Utah, Salt Lake City, Utah, USA \\ Address correspondence to: Jeffrey E. DeClue, Laboratory of Cellular Oncology, National Cancer Institute, \\ Building 36, Room 1D-32, Bethesda, Maryland 20892, USA. \\ Phone: (301) 496-4732; Fax: (301) 480-5322; E-mail: jd99f@nih.gov. \\ Giovanna Benvenuto's present address is: Department of Cellular and Molecular Biology and Pathology, \\ Faculty of Medicine, University of Naples, Naples, Italy. \\ Received for publication June 16, 1999, and accepted in revised form March 20, 2000.
}

\begin{abstract}
We have found that EGF-R expression is associated with the development of the Schwann cell-derived tumors characteristic of neurofibromatosis type 1 (NF1) and in animal models of this disease. This is surprising, because Schwann cells normally lack EGF-R and respond to ligands other than EGF. Nevertheless, immunoblotting, Northern analysis, and immunohistochemistry revealed that each of 3 malignant peripheral nerve sheath tumor (MPNST) cell lines from NF1 patients expressed the EGF$\mathrm{R}$, as did 7 of 7 other primary MPNSTs, a non-NF1 MPNST cell line, and the $\mathrm{S}^{100^{+}}$cells from each of 9 benign neurofibromas. Furthermore, transformed derivatives of Schwann cells from $\mathrm{NF}^{-/-}$mouse embryos also expressed the EGF-R. All of the cells or cell lines expressing EGF-R responded to EGF by activation of downstream signaling pathways. Thus, EGF-R expression may play an important role in NF1 tumorigenesis and Schwann cell transformation. Consistent with this hypothesis, growth of NF1 MPNST lines and the transformed $\mathrm{NF}^{-/-}$mouse embryo Schwann cells was greatly stimulated by EGF in vitro and could be blocked by agents that antagonize EGF-R function.
\end{abstract}

J. Clin. Invest. 105:1233-1241 (2000).

\section{Introduction}

Neurofibromatosis type 1 (NF1) is a dominantly inherited human disease affecting one in 2,500 to 3,500 individuals $(1,2)$. The NF1 phenotype is highly variable, and its clinical course is unpredictable. Several organ systems are affected, including the bones, skin, iris, and central nervous system (manifested in learning disabilities and gliomas) (3). The hallmark of NF1 is the development of benign tumors of the peripheral nervous system (neurofibromas), which vary greatly in both number and size among patients $(1,4)$. Neurofibromas are heterogeneous tumors composed of Schwann cells, neurons, fibroblasts and other cells, with Schwann cells being the major $(60-80 \%)$ cell type $(5,6)$. Neurofibroma-derived Schwann cells have been demonstrated to possess abnormal properties, including increased invasiveness and the induction of angiogenesis (7). NF1 patients are also at increased risk for the development of certain malignancies, including pheochromocytomas, childhood myeloid leukemias, and in about 5\% of patients, malignant peripheral nerve sheath tumors (MPNST) (8-10). Although still a point of some debate, it is widely held that MPNST arise from large (plexi- form) neurofibromas and are probably derived from Schwann cells, because many MPNST stain positive for Schwann cell markers such as $\mathrm{S} 100(4,11)$.

The NF1 gene lies on chromosome 17 (12-14), and the great majority of patient mutations prevent expression of the intact NF1 product, designated neurofibromin (15). Neurofibromin contains a central domain homologous to a family of proteins known as Ras-GTPase-activating proteins (Ras-GAPs), which function as negative regulators for Ras proteins (16). Ras-GAPs attenuate signaling from Ras, thus blocking the transmission of signals leading to increased growth or differentiation. The role of neurofibromin as a tumor suppressor that inactivates Ras-dependent signals has been confirmed. Primary neurofibromas and cell lines derived from NF1 MPNST show high levels of Ras-GTP, and in MPNST cell lines lacking neurofibromin, elevated Ras-GTP leads to constitutive growth activation (17-19).

Targeted disruption of NF1 in mice has provided an experimental system for analyzing the role of neurofibromin in growth regulation $(20,21)$. The importance of Schwann cells to tumor development in NF1 is sug- 
gested by the altered properties of Schwann cells from heterozygous $(+/-)$ or homozygous mutant (-/-) embryos (22). Mutant Schwann cells have elevated RasGTP, are more invasive than wild-type cells, and when cultured in low serum yield transformed derivatives (TXF) that display altered morphology, reduced growth factor dependence, and reduced adherence (23). These transformed derivatives, which fail to develop in cultures of wild-type $(+/+)$ littermates, retain expression of the Schwann cell markers P75 and S100 (23).

Schwann cell growth in vivo is normally regulated through interactions with neurons (4). The family of small peptides known as heregulins/neuregulins, including glial growth factor (GGF), probably serve as in vivo Schwann cell mitogens. Neuregulins, which robustly stimulate Schwann cell growth in vitro (24), bear homology to EGF and activate transmembrane tyrosine kinase receptors (erbB2, -3 , and -4 ) that are structurally and functionally related to the EGF-R (reviewed in ref. 25). Schwann cells, which express little if any erbB4, use erbB2-3 heterodimers for GGF signaling (26). Stimulation of Schwann cells with GGF in vitro leads to increases in Ras-GTP, demonstrating a link between Ras regulation and Schwann cell proliferation (27).

Both NF1 alleles are disrupted in MPNST and in at least a proportion of benign neurofibromas (28-30). Whereas mutation or loss of the $p 53$ gene occurs in about one third of MPNST (31), it is likely that additional alterations are present in these tumors. Here we report evidence indicating that aberrant expression of the EGF-R is associated with tumor development in NF1 and in animal models of NF1, suggesting a role in pathogenesis and representing a novel potential therapeutic target.

\section{Methods}

Cell culture and biochemical assays. MPNST lines were grown as described (17), and the embryo-derived mouse Schwann cells and TXF derivatives were isolated and grown as described $(22,23)$. Agar colony formation assays were carried out as described (17). For MAP kinase assays, cells were grown until confluent, serumstarved for 24 hours, stimulated with mitogen for 5 minutes at $37^{\circ} \mathrm{C}$, then lysed, and MAP kinase assays were carried out as described (23). For Western blotting, cells were grown until confluent and lysed. Lysates with $50 \mu \mathrm{g}$ protein (human) or $100 \mu \mathrm{g}$ protein (mouse) were subjected to SDS-PAGE using $6 \%$ gels. Immunoblotting was carried out as described (17) using the following antibodies from Santa Cruz Biotechnology (Santa Cruz, California, USA): for EGF-R, sc03; for erbB2, sc284; for erbB3, sc285; for erbB4, sc283. Antibodies were used at a dilution of 1:2,000 for human and 1:1,000 for mouse lysates. Blots were developed with an enhanced chemiluminescence detection kit (Kirkegaard \& Perry Laboratories, Gaithersburg, Maryland, USA).

Northern blotting. Cells were grown until confluent and lysed, and total RNA was extracted using the RNeasy system (QIAGEN Inc., Valencia, California, USA). Twenty micrograms of total RNA from each line was electrophoresed in an agarose gel and transferred to a nylon filter (Millipore Corp., Bedford, Massachusetts, USA). Human EGF-R probe was derived from the plasmid pCO12 (32) by digestion with SstII and XhoI. The probe was labeled with ${ }^{32} \mathrm{P}$ in a nick translation system (Promega Corp., Madison, Wisconsin, USA), and hybridization was carried out using Quickhyb buffer (Stratagene, La Jolla, California, USA) with $1.4 \times 10^{7}$ cpm of probe.

Immunohistochemistry. All immunohistochemistry was performed on 4-6-micron paraffin sections using the Ventana ES immunostaining system (Ventana Instruments, Tuscon, Arizona, USA). Following deparaffinization in xylenes and trypsinization (for EGF-R detection), slides were placed in the instrument that adds the primary antibody, the biotinylated anti-mouse or rabbit second antibody, and avidin-conjugated peroxidase or alkaline phosphatase as dictated by a bar code. Primary antibodies were incubated for $32 \mathrm{~min}-$ utes at $37^{\circ} \mathrm{C}$. The instrument performed all washes. Primary antibodies were rabbit polyclonal anti-bovine S100 (Dakopatts Inc., Carpinteria, California, USA) diluted to 1:1000; mouse monoclonal anti-EGF-R 31G7 (1:8; Zymed Inc., South San Francisco, California, USA); or PG-M1, a mouse monoclonal antimacrophage marker (Dakopatts) diluted to 1:100. Slides were counterstained with hematoxylin or with nuclear fast red by hand. In all cases irrelevant mouse or rabbit immunoglobulin was used instead of a primary antibody as a negative control.

Dissociated cell preparations. Normal human nerves $(n=2)$ and neurofibroma $(n=3)$ specimens were digested overnight in enzymes as described (6). Cells $\left(10^{6}\right)$ were washed twice in L15 medium (GIBCO/BRL, Grand Island, New York, USA) then fixed in nonbuffered formalin, pelleted, and centrifuged into a cassette (Cytoblock; Shandon Inc., Pittsburgh, Pennsylvania, USA) for embedding.

Patient information. Adult normal nerves were used as controls. When NF1 diagnosis (NF1 dx) was made, it was based on family history, café-au-lait macules, and neurofibromas as described (33). Cutaneous neurofibromas were obtained from: 69-year-old man (non-NF1); 50year-old woman (non-NF1); adult (non-NF1); 30-yearold woman (NF1). Plexiform neurofibromas were obtained from: 17-year-old man (NF1 dx); 6-year-old boy (NF1 dx); 15-year-old girl (NF1 dx); 14-year-old girl (NF1 $\mathrm{dx}$ ); and a patient (mid-20s) with segmental NF1. Neurofibromas used for cell dissociation were obtained from: adult woman, cutaneous neurofibroma (NF1 dx); 3-year-old child, cervical neurofibroma (NF1 dx). For MPNST used see Table 1.

\section{Results}

NF1 patient tumor lines respond to EGF and express EGF-R. Stimulation of primary Schwann cells with GGF (10 $\mathrm{ng} / \mathrm{mL}$ ) led to a dramatic (approximately 80 -fold) activation of mitogen-activated protein kinase (MAP kinase) (Figure 1a), consistent with the role of GGF as 
a major Schwann cell mitogen, whereas EGF (50 $\mathrm{ng} / \mathrm{mL}$ ) had no effect. Compared with primary Schwann cells, the basal level of MAP kinase in serumstarved cells was substantially elevated (5-12-fold) in NF1 patient MPNST-derived cell lines (Figure 1a). Surprisingly, treatment of these lines with GGF failed to elicit activation of MAP kinase in two of three lines tested (lines 90-8 and 88-14), whereas only the 88-3 line responded to GGF (three- to fourfold). In contrast to primary Schwann cells, treatment of all three MPNST lines with EGF led to MAP kinase activation (six- to ninefold) (Figure 1a). These results suggested that the NF1 patient lines express the EGF receptor, but only the 88-3 line expresses the GGF receptor. To analyze directly the expression of receptors for EGF and GGF in these cells, we prepared lysates from growing cells and probed them by immunoblotting with antibodies specific for EGF-R and for erbB2, -3 , and -4 (Figure 1b). The 88-3, 90-8, and 88-14 lines all expressed a strong band at approximately $170 \mathrm{kDa}$ corresponding to EGF-R and also expressed abundant levels of erbB2, whereas only the 88-3 line expressed
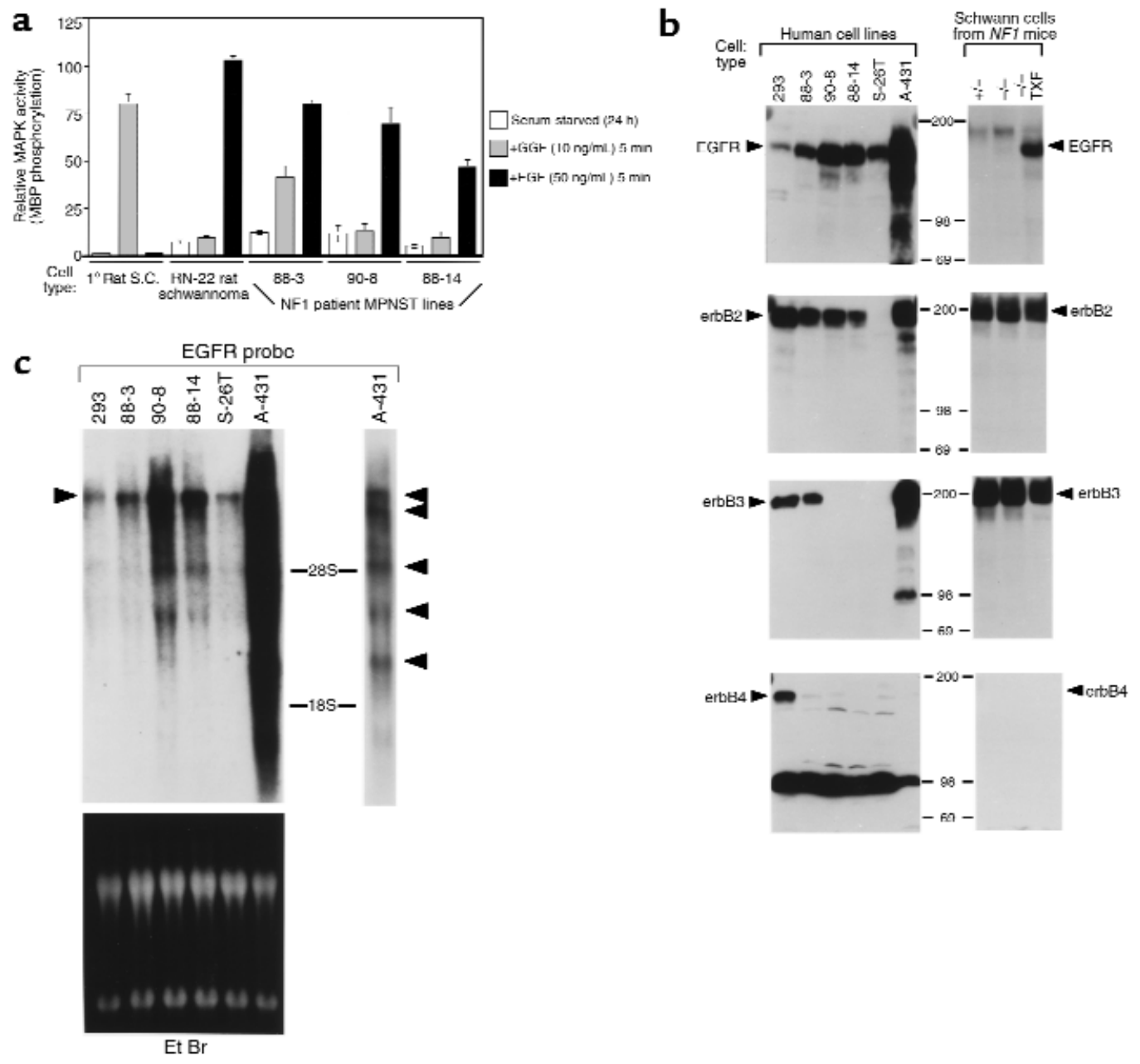

Figure 1

Response of primary rat Schwann cells, RN-22 rat schwannoma line, and human NF1 patient MPNST lines to GGF and EGF, and expression of EGF-R and erbB2, -3 , and -4 proteins and EGFR mRNA. (a) The indicated cells were grown until nearly confluent, serum starved for 24 hours, then left untreated (-) or stimulated with $10 \mathrm{ng} / \mathrm{mL}$ recombinant human GGF (G) or $50 \mathrm{ng} / \mathrm{mL}$ recombinant human EGF (E) for 5 minutes at $37^{\circ} \mathrm{C}$. The cells were lysed and the endogenous MAP kinase activity was assayed. Following the reaction, incorporation of ${ }^{32} \mathrm{P}_{\mathrm{i}}$ into exogenous myelin basic protein was determined. Values were normalized to unstimulated primary rat Schwann cells (1.0) and represent the results of two experiments, carried out in duplicate, with error bars shown. (b) Expression of EGF-R and erbB2, -3, and -4 proteins in human and mouse cells. Cells were grown until confluent and lysed, and lysates containing $50 \mu \mathrm{g}$ human or $100 \mu \mathrm{g}$ mouse cell protein were subjected to analysis by SDS-PAGE and immunoblotting using antibodies specific for each protein indicated (arrows). 293, human embryonic kidney cells; 88-3, 90-8, 88-14, human NF1 MPNST lines; S-26T, human non-NF1 MPNST line; A-431, human epidermoid carcinoma line. Migration of molecular standards $(\mathrm{kDa})$ is indicated at center. A strong nonspecific band of approximately $90 \mathrm{kDa}$ appeared in the erbB4 blot of human but not mouse lysates. (c) Expression of EGFR mRNA in human MPNST and control cell lines. Cells were grown until confluent and lysed, and $20 \mu \mathrm{g}$ of total RNA from each line was subjected to electrophoresis, transferred to a filter, and hybridized to a human EGFR probe labeled with ${ }^{32} \mathrm{P}$. The predominant $10.5-\mathrm{kb} \mathrm{mRNA}$ is indicated with an arrow at left, as is the approximate location of the $28 \mathrm{~S}$ and $18 \mathrm{~S}$ RNAs (top). At right is a shorter exposure of the A-431 line, with arrows designating the different mRNAs detected. The filter was photographed under ultraviolet light before hybridization (bottom). 
erbB3. None of the NF1 patient lines expressed significant levels of erbB4. The S-26T line is a $\mathrm{S} 100^{+}$line from a non-NF1 patient (34) that displayed EGFresponsive MAP kinase activation, but this line failed to respond to GGF (G. Benvenuto and J.E. DeClue, unpublished data). This line expressed EGF-R but not erbB2, -3 , or -4 (Figure 1b). Control lines included human embryonic kidney cells (line 293), which expressed all four proteins, and A-431 carcinoma cells, which contain amplified and rearranged copies of the EGFR gene (35) and expressed very high levels of EGF$\mathrm{R}$ and smaller, related peptides, as well as erbB2 and -3 . EGF treatment of all four MPNST lines yielded a $170-\mathrm{kDa}$ tyrosine-phosphorylated band corresponding to the EGF-R, whereas GGF treatment led to the appearance of a 180-190 kDa tyrosine-phosphorylated erbB3 band in lysates from primary Schwann cells and the 88-3 cell line, but not in lysates from the 8814, 90-8, or S26-T lines (data not shown). We conclude that human NF1 MPNST lines express EGF-R and respond to EGF, whereas only one of the lines expresses erbB3 and responds to GGF. To confirm the expression of EGF-R in these lines at the level of messenger RNA, we carried out Northern analysis using a human EGFR-specific probe (Figure 1c). A specific mRNA corresponding to the $10.5-\mathrm{kb}$, full-length $E G F R$ mRNA (36) was detected in each of the MPNST lines (left arrow), as well as in 293, and the intensity of the band provided a striking parallel to the immunoblotting data for EGF-R in Figure 1b. A-431 cells expressed a variety of forms as described previously, including the 10.5-kb band (right panel; top arrow) and a $2.9-\mathrm{kb}$ mRNA resulting from a rearranged copy of the gene (bottom arrow) (35). Based on these results, it is clear that the MPNST lines contain both EGF-R mRNA and protein and display a strong correlation between the levels of mRNA and protein expressed.

The ethylnitrosourea-induced rat schwannoma cell line RN-22, which expresses the Schwann cell marker S100 and normal levels of the neurofibromin, and likely contains a mutation in the neu gene encoding rat erbB2, was also analyzed in these experiments $(25,37)$. Similar to the human NF1 MPNST lines, the elevated basal level of MAP kinase activity in these cells was dramatically stimulated (15-fold) by EGF, whereas GGF had no effect (Figure 1a). Direct immunoblotting of RN-22 lysates with anti-phosphotyrosine antiserum revealed a $170-\mathrm{kDa}$, EGF-stimulated band, as well as a constitutive 185-kDa band (erbB2) (data not shown). Thus, an alternative route of Schwann cell tumorigenesis in a different species also led to expression of the EGF-R and loss of GGF responsiveness.

Primary benign and malignant NF1 patient tumors express $E G F-R$. The experiments described above suggest that one of the events leading to malignant tumorigenesis in NF1 is the acquisition of EGF-R expression. To test whether EGF receptors are expressed in primary tumors associated with NF1 disease, and not just in

Table 1

MPNST sections analyzed for EGF-R and S100 expression

$\begin{array}{lc}\text { Patient information } & \begin{array}{c}\text { EGF-R expression } \\ \text { MPNST: 14-year-old girl; }\end{array} \\ \begin{array}{l}\text { NF1 status unknown } \\ \text { robustly EGF-R-positive: } \\ \end{array} & \begin{array}{c}\text { other areas EGF-R-negative } \\ \text { MPNST: 42-year-old woman; }\end{array} \\ \text { NF1 status unknown (Figure 2, a and b) } & \begin{array}{c}\text { Uniform moderate EGF-R staining } \\ \text { in at least 70\% of tumor cells }\end{array}\end{array}$

NF1 status unknown (Figure 2, a and b)

in at least $70 \%$ of tumor cells

Multiple foci robustly EGF-R-positive cells

MPNST: 31-year-old woman;

NF1 status unknown

MPNST/Triton tumor:

13-year-old boy; NF1 dx

MPNST: adolescent

Non-NF1

MPNST: 44-year-old man;

NF1 dx

MPNST: 30-year-old woman; NF1 dx (Figure2, c and d)
Robustly EGF-R-positive

Weak EGF-R-positive staining in up to $10 \%$ of cells

Uniform moderate EGF-R-positive staining throughout

Very rare islands of weak EGF-R-positive staining: majority of tumor is negative

\section{S100 $\beta$ expression \\ S100-negative}

Scattered robustly S100-postive cells (may be macrophages); other areas patch weak S100-positive; some areas S100-negative

S100-negative

$$
\begin{aligned}
& \text { S100-negative to uniform trace } \\
& \text { S100-positive in } 1 / 3 \text { of tumor } \\
& \text { Robust S100-positive staining } \\
& \text { most or all tumor cells } \\
& \text { S100-negative to uniform trace } \\
& \text { S100-positive in patches }
\end{aligned}
$$

Strongly positive throughout

Immunohistochemistry was carried out as described in Methods. Two individuals read each slide (S. Heffelfinger and N. Ratner). In total, only one of seven tumors was almost entirely EGF-R-negative, and even that tumor may have some positive regions. For S100, three of seven are positive, two of seven are negative, and two of seven show trace or no expression. dx: NF1 diagnosis; non-NF1, failed to meet consensus criteria and no evidence of NF1 mutation. 
cultured cell lines, we immunostained sections from normal human nerves, benign neurofibromas, and MPNST with anti-EGF-R or anti-S100. Seven primary MPNST specimens were analyzed for expression of S100 and EGF-R. All seven tumors showed some EGF$\mathrm{R}$ expression, but a range of expression patterns was evident (Table 1). Some tumors showed robust staining in nearly all cells, whereas others contained only rare, weakly positive cells. For example, sections from a tumor in which the majority of the cells showed strong EGF-R immunoreactivity is shown in Figure $2 \mathrm{~b}$. This tumor was negative for expression of S100 (Figure 2a). The other extreme example is shown in Figure 2, c and $\mathrm{d}$. The majority of cells in this tumor were negative for EGF-R expression (Figure 2d), whereas many cells were positive for S100 expression (Figure 2c). In another case, focal areas within a single MPNST section had robust EGF-R immunoreactivity, whereas neighboring areas in the same section were negative (data not shown). We conclude that primary MPNST contain varying proportions of cells that express the EGF-R, with some tumors expressing high levels of EGF-R in most of their cells. These results reinforce the findings described above for the MPNST-derived cell lines.

In normal nerves, $\mathrm{S} 100^{+}$cells are found only in the endoneurial compartment (Figure 2e), whereas EGF-R ${ }^{+}$ cells (fibroblasts) make up the perineurium (Figure 2f). Sections from nine neurofibromas were analyzed, four from cutaneous neurofibromas, and five from the much larger plexiform neurofibromas. All showed both $\mathrm{S} 100^{+}$cells (Schwann cells) and distinct EGF-R ${ }^{+}$cells (likely fibroblasts) (Figure $2 \mathrm{~g}$ ). In nine of nine neurofibroma specimens, occasional cells appeared to express both antigens. To verify this, we dissociated cells from three additional neurofibromas, isolated the cells, and embedded them in paraffin. Staining sections with anti-S100 and anti-EGF-R demonstrated a population of $\mathrm{S}_{100} / \mathrm{EGF}^{+} \mathrm{R}^{+}$cells (Figure $2 \mathrm{~h}$ ). We found that $1.8 \%$ of cells expressed both antigens $(6 / 190,1 / 110$, and $3 / 216$ double-labeled cells in three independent counts). In contrast, normal nerve samples completely lacked double-labeled cells and contained 99\% S100+ Schwann cells, and $1 \%$ EGF-R ${ }^{+}$cells (fibroblasts/perineurial cells) (Figure 2i). When sections were stained with an anti-macrophage marker (PG-M1) and antiS100, no double-labeled cells were observed (not shown). We conclude that certain cells in neurofibromas, most likely Schwann cells, express both S100 and EGF-R, whereas such cells are absent in normal nerves.

In vitro transformation of mouse NF1-deficient Schwann cells leads to EGF-R expression. Mice with targeted mutations in the NF1 gene represent a model system for investigating NF1 tumorigenesis $(20,21)$. Although homozygous mutant (-/-) embryos die by day 14.5 , we have developed techniques to purify Schwann cells from day12.5 embryos $(22,23)$. To investigate MAP kinase signaling, these Schwann cells were serum starved, stimulated with GGF, and lysed (Figure 3a). Although Schwann cells lacking one $(+/-)$ or both $(-/-)$ copies of
NF1 have elevated Ras-GTP, there was little or no enhancement of basal MAP kinase activity compared with wild-type cells (Figure 3a). Cells of all genotypes responded to GGF stimulation with large increases in MAP kinase activity. The TXF Schwann cells isolated from (-/-) embryos displayed an increased basal level of MAP kinase activity and responded to GGF.

We hypothesized that the in vitro transformation of NF1-deficient mouse Schwann cells might be associated with EGF-R expression. To test this, we examined MAP kinase activity following EGF stimulation of serumstarved (-/-) or TXF cells (Figure 3b). Whereas EGF elicit-
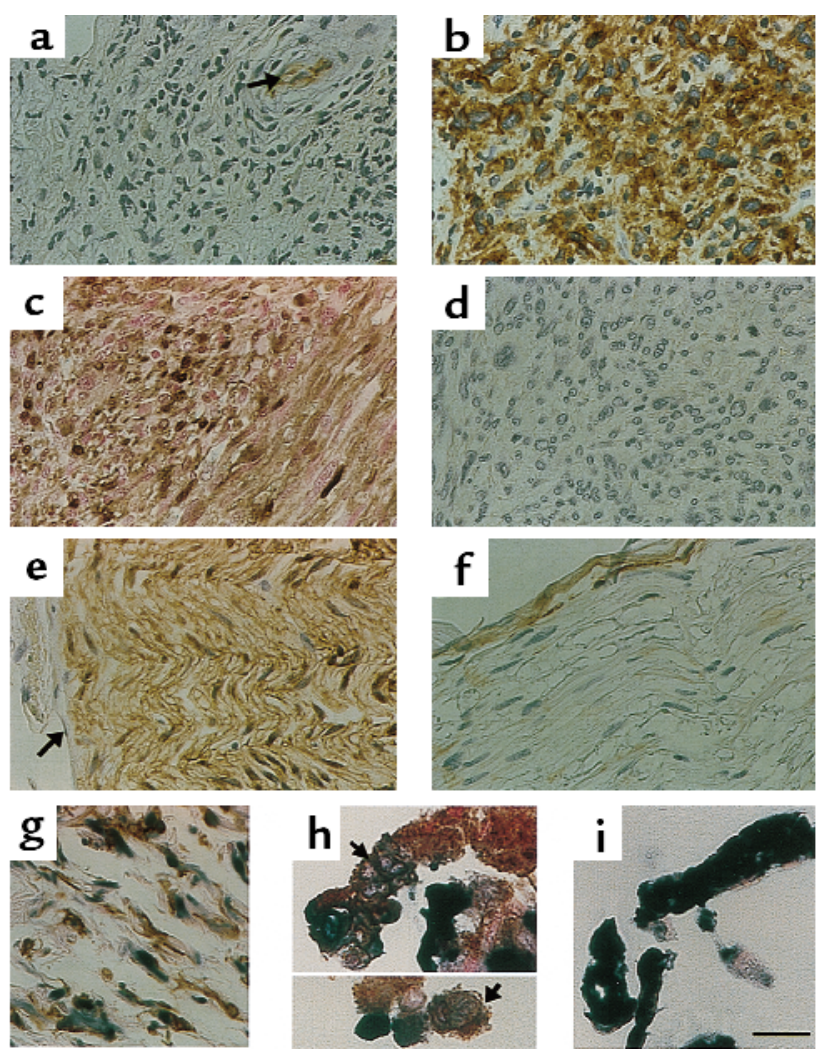

Figure 2

EGF-R expression in NF1 tumor sections. Paraffin sections were stained with anti-EGF-R (b, d, and $\mathbf{f}$ ) or anti-S100 (a, c, and $\mathbf{e})$ or with both antibodies $(\mathbf{g}, \mathbf{h}$, and $\mathbf{i})$. Visualization of single antibodies was with 3'3-diaminobenzidine $\mathrm{HCl}$ (DAB; brown) (a-f). Double labeling used nitroblue tetrazolium (NBT/BCIP; blue) for anti$\mathrm{S} 100$, and DAB (brown) for anti-EGF-R ( $\mathbf{g}-\mathbf{i})$. In $\mathbf{a}, \mathbf{b}$, and $\mathbf{d}-\mathbf{f}$, the counterstain is hematoxylin (blue); in $\mathbf{c}$ and $\mathbf{g}-\mathbf{i}$, the counterstain is nuclear fast red (pink). (a-d) Sections from MPNST. In a, the arrow points to a normal nerve within the tumor, containing $\mathrm{S}_{100}{ }^{+}$cells, whereas the tumor matrix is $\mathrm{S}_{100^{-}}$. An adjacent section from the same tumor in $\mathbf{b}$ shows that most cells are EGF- $\mathrm{R}^{+}$. Another MPNST contains $\mathrm{S}_{100}{ }^{+}$cells (c) and is mostly negative for EGF-R (d). (e and $\mathbf{f}$ ) Sections of normal human nerve (arrow points to perineurium). (g) A section of a cutaneous neurofibroma with EGF$\mathrm{R}^{+} / \mathrm{S} 100^{+}$cells.(h) A section from a dissociated neurofibroma cell preparation. The arrow designates a group of cells double stained by anti-S100 and anti-EGF-R. (i) A section from a dissociated normal nerve preparation. No double-labeled cells are detected. $\mathbf{a - g}$, bar: $34.2 \mu \mathrm{m}$; $\mathbf{h}$ and $\mathbf{i}$, bar: $16.3 \mu \mathrm{m}$. 
ed no increase in MAP kinase activity in the (-/-) cells, TXF derivatives displayed a dramatic increase, even greater than the response to GGF. Schwann cells from wild-type (+/+) or heterozygous (+/-) embryos failed to respond to EGF (data not shown). To examine the expression of EGF-R and erbB2, -3, and -4, we carried out immunoblotting with antibodies against these proteins (Figure $1 \mathrm{~b})$. Lysates of $(+/+)$ and $(-/-)$ cells expressed erbB2 and -3, but not EGF-R. In contrast, the (-/-) TXF cells expressed abundant levels of EGF-R, as well as erbB2 and -3 . None of the cells expressed erbB4, consistent with studies published previously (26). Immunoblotting of the lysates from Figure $3 \mathrm{~b}$ confirmed the activation of EGF-R in the (-/-) TXF cells following EGF treatment, as well as tyrosine phosphorylation of erbB3 in GGFstimulated lysates from TXF and (-/-) cells (data not shown). We conclude that acquisition of EGF-R expression is associated with in vitro transformation of NF1deficient Schwann cells. These results represent a striking parallel to the results obtained from our analysis of human NF1-related tumors.
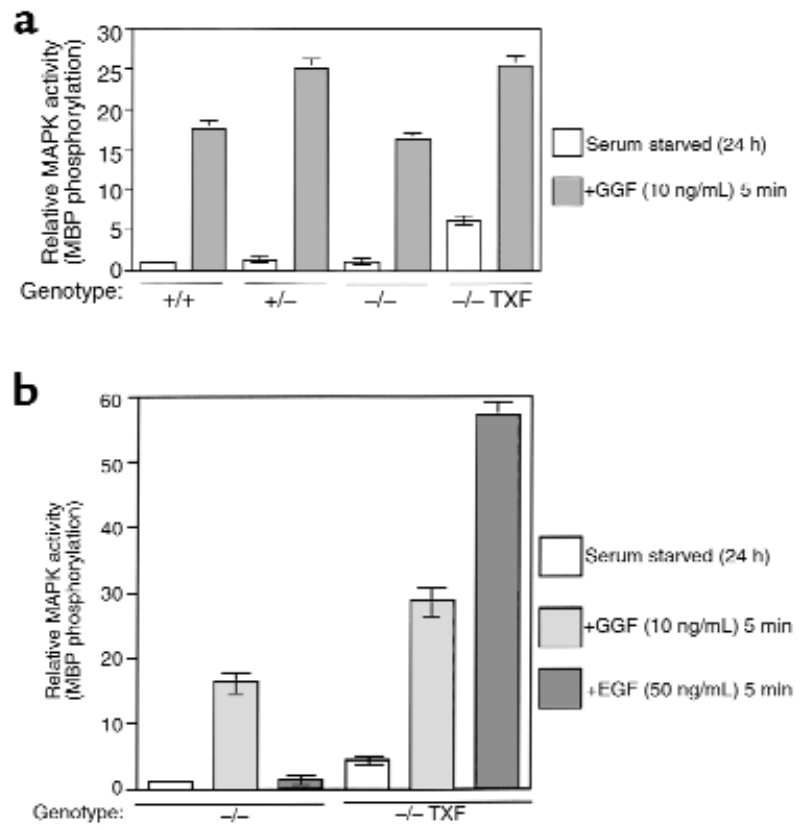

\section{Figure 3}

Transformed derivatives of NF1 (-/-) mouse embryo-derived Schwann cells have elevated basal MAP kinase activity and respond to EGF. (a) Mouse Schwann cells were isolated from day 12.5 embryos with wildtype NF1 $(+/+)$ or targeted disruption of one NF1 allele (+/-), or both alleles $(-/-)$, and compared with transformed derivatives of $(-/-)$ (TXF). The cells were serum starved for 24 hours, then left untreated or stimulated with GGF, as indicated. Cells were lysed and MAP kinase activity was determined as for Figure 1. Results are the mean of two experiments carried out in duplicate, with error bars shown. Results were normalized to the level of activity present in serum-starved wildtype $(+/+)$ cells (1.0). (b) Schwann cells from (-/-) embryos and TXF were treated and assayed as above, except additional samples were prepared after stimulation with $50 \mathrm{ng} / \mathrm{mL}$ EGF for 5 minutes. Results are the mean of two experiments carried out in duplicate, with error bars shown. Results were normalized to the level of activity present in serum-starved homozygous null (-/-) cells (1.0).
Growth of NF1 patient tumor lines is EGF dependent and can be blocked by EGF-R antagonists. We wished to determine whether expression of the EGF-R in NF1 tumors and cell lines affected the growth of these cells. When the 8814 cell line was grown in limiting amounts of FBS $(0.1 \%)$, cells lacking EGF remained viable (as judged by trypan blue exclusion and replating under growth conditions) but did not grow, whereas cells treated with 10 $\mathrm{ng} / \mathrm{mL}$ EGF grew at a rapid rate (Figure 4a). The EGFdependent growth of the 88-14 line suggested that blocking the EGF-R might inhibit the growth of the cells. Therefore, we tested EGF-R antagonists for their ability to suppress growth of 88-14 cells under the same conditions (Figure 4a). Treatment with $\mathrm{mAb} 225$, an antibody that blocks EGF-R activation (38), significantly inhibited cell growth, whereas even more dramatic inhibition was achieved with chemical inhibitors of EGF-R tyrosine kinase activity, the tyrphostins A-25 and AG-1478 (39). All of the EGF-R antagonists displayed significantly greater inhibition than that observed for the potent farnesyltransferase inhibitor B581 (40). Whereas previous studies demonstrated that farnesyltransferase inhibitors slow the growth of the 8814 line in vitro, most likely by blocking Ras or RhoB activity (41), these results demonstrate that antagonizing the EGF-R also can block their growth.

As an additional test for these agents to antagonize EGF-dependent growth of the 88-14 tumor line, we carried out assays of anchorage-independent growth in soft agar (Figure $4 \mathrm{~b}$ ). When plated in moderate levels (7\%) of serum, no significant growth was observed after 4 weeks. However, the presence of $100 \mathrm{ng} / \mathrm{mL}$ EGF supported the formation of small- to medium-sized colonies under these conditions (Figure $4 \mathrm{~b}$ ). Furthermore, the presence of $\mathrm{mAb} 225$ or tyrphostin AG-1478 strongly inhibited the formation of such colonies (Figure $4 \mathrm{~b}$ ), whereas B581 had no significant effect (data not shown). We conclude that under limiting conditions, the growth of 88-14 is dependent on EGF and can be blocked by EGF-R antagonists. In other experiments, we found that the addition of EGF dramatically stimulated the growth of (-/-) TXF cells in vitro, and that tyrphostin A-25 could block this effect (data not shown). In contrast, EGF had no effect on nontransformed (-/-) Schwann cells or on Schwann cells from $(+/-)$ or $(+/+)$ embryos (data not shown). We conclude that EGF-R expression has a significant biological effect on the growth of both NF1 patient tumorderived cells and TXF cells from NF1-deficient mice.

The experiments just described were carried out in conditions where exogenous EGF was essential for the growth of the cells. Whereas the availability of EGFrelated peptides and other growth factors in the tumor environment is unknown, we nevertheless wished to determine the effect of EGF-R antagonists on the growth of NF1 patient lines under other growth conditions. Therefore, we examined the growth of the 8814 and the 90-8 MPNST lines in the presence of $2 \%$ FBS with no EGF added (Figure 4, $c$ and d). Whereas 
a

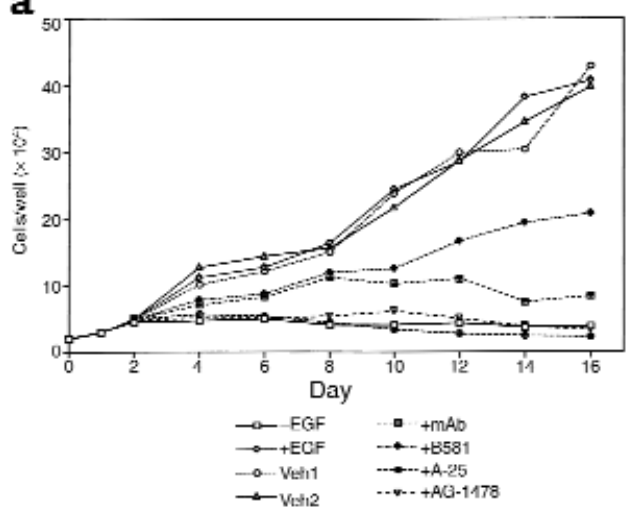

c

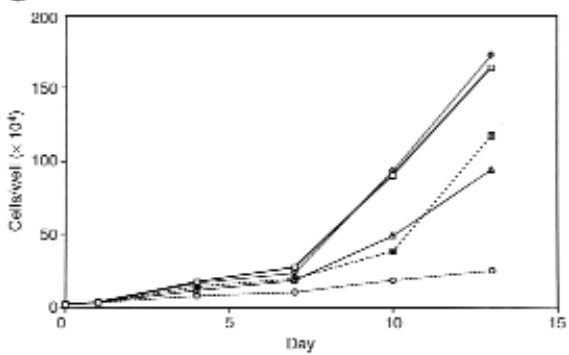

b

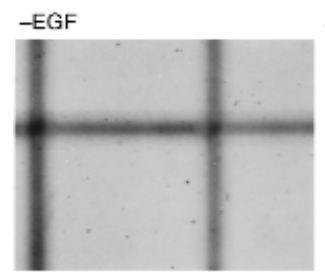

$\pm \mathrm{EGF} / \mathrm{mAb} 225$

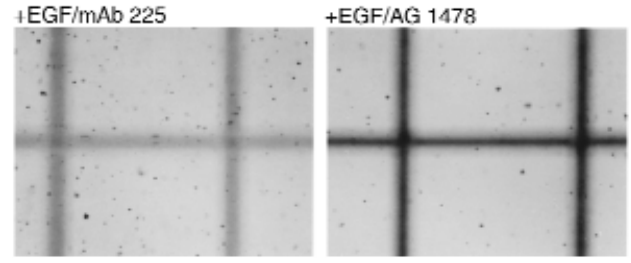

d

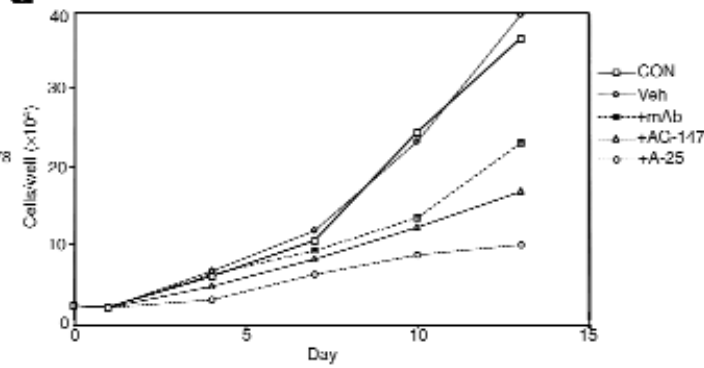

\section{Figure 4}

Growth of NF1 patient MPNST lines is EGF dependent and is inhibited by EGF-R antagonists. (a) MPNST line $88-14$ was plated at $2 \times 10^{4}$ per 35-mm well. The next day (day 1 ) cells were switched to medium without (-EGF) or with $10 \mathrm{ng} / \mathrm{mL}$ EGF (+EGF), and on day 2 cells were switched to medium containing $10 \mathrm{ng} / \mathrm{mL}$ EGF and 3\% PBS (Veh1); 0.1\% DMSO (Veh2); $3 \mu \mathrm{g} / \mathrm{mL} \mathrm{mAb} 225 \mathrm{in} \mathrm{PBS} \mathrm{(3 \%} \mathrm{vol/vol;} \mathrm{mAb);} 40$ $\mu \mathrm{M}$ farnesyltransferase inhibitor B581 in water; $10 \mu \mathrm{M}$ tyrphostin A-25 in DMSO; or $400 \mathrm{nM}$ tyrphostin AG-1478, also in DMSO. Cells were re-fed with medium containing fresh inhibitor and counted in duplicate every 2 days, beginning at day 2. (b) Growth of MPNST 88-14 line in soft agar. Cells were plated at $10^{5} / \mathrm{mL}$ in a $0.4 \%$ (wt/vol) agar suspension with $7 \%$ FBS with or without $100 \mathrm{ng} / \mathrm{mL}$ EGF, as indicated; mAb 225 was included at $5 \mu \mathrm{g} / \mathrm{mL}$ and AG-1478 at $400 \mathrm{nM}$. Cells were photographed after 4 weeks. $\times 25$. (c and d) Growth of 88-14 (c) and 908 (d) in $2 \%$ serum is inhibited by EGF-R antagonists. Cells were plated as in a, and switched on day 1 to medium containing $2 \%$ FBS (CON) or $2 \%$ serum plus $0.1 \%$ DMSO (Veh); $3 \mu \mathrm{g} / \mathrm{mL}$ mAb 225 (mAb); $10 \mu \mathrm{M}$ tyrphostin A-25; or $400 \mathrm{nM}$ tyrphostin AG-1478. Thereafter, cells were re-fed with medium containing fresh inhibitor every 2 days, and duplicate wells of cells were counted every three days.

the 88-14 line grew much more robustly than 90-8 under these conditions (Figure 4, $\mathrm{c}$ and $\mathrm{d}$ ), the growth of both lines was significantly inhibited by the addition of tyrphostin A-25 and to a lesser extent by tyrphostin AG-1478. The anti-EGF-R mAb also inhibited the growth of these lines in serum, although the effect was somewhat less pronounced (Figure 4, c and d). These results demonstrate that EGF-R expression affects the growth of tumor cell lines derived from NF1 patients under conditions where EGF is not the primary factor driving growth of the cells.

\section{Discussion}

Here we have described aberrant expression of the EGF$\mathrm{R}$ in primary tumors from patients with NF1, in cell lines derived from those tumors, in a cell line from a non-NF1 patient with MPNST, in a chemically induced rat schwannoma line, and in in vitro-transformed Schwann cells from mouse embryos lacking an intact NF1 gene. The congruence of the animal model systems with the results from NF1 patient materials suggests that aberrant expression of the EGF-R might play an important role in the development of peripheral nerve tumors in NF1 patients and that mutation of NF1 may predispose the tumor progenitor cells to express EGFR. In the cells tested, we found a perfect correlation between expression of EGF-R and EGF responsiveness and also between expression of erbB2/erbB3 and GGF responsiveness. Additionally, the level of EGF-R mRNA in the human MPNST lines correlated well with the level of protein expression. There is a strong precedent for involvement by the EGF-R and other members of the erbB family in human cancer, because over $60 \%$ of all solid tumors overexpress at least one of these proteins or their ligands (reviewed in refs. 42 and 43). Furthermore, a negative correlation has been demonstrated between EGF-R expression and survival in certain cancers and with progression to malignancy in others (43). We have found that the growth of cell lines derived from MPNSTs and transformed mouse Schwann cells in vitro was highly EGF dependent and could be blocked by EGF-R antagonists under conditions where EGF was the primary growth factor. This demonstrates that the expressed EGF-R is capable of transmitting 
mitogenic signals in these cells. We also found that EGF-R antagonists could block the growth of MPNST lines growing in serum without added EGF. This inhibition may be due to the blocking of potential autocrine growth stimulation pathways in the cells; alternatively, it may indicate that the EGF-R is involved (directly or indirectly) in mitogenic signaling by serum factors that induce growth of the cells.

Other than mutation or loss of the second (normal) NF1 allele (10), little is known of the specific genetic and epigenetic events involved in the development of NF1-related neurofibromas and MPNSTs. Earlier work suggested a possible involvement of $p 53$ in NF1-related MPNST formation (31), and it was recently shown that p53 and NF1 cooperate in tumor formation in mice (44, $45)$. Furthermore, the hypothesis of a Schwann cell origin for both benign neurofibromas and MPNSTs remains to be proven. However, our data support other studies suggesting that deregulation of Schwann cell growth is a primary defect driving the development of both benign neurofibromas and MPNST in NF1 patients (4). The immunohistochemical analyses revealed that at some point during or after the formation of neurofibromas, a subset of the $\left(\mathrm{S} 100^{+}\right)$Schwann cells acquire EGF-R expression. Such cells were not observed in normal control nerves, although a previous analysis of tissue sections of benign schwannomas from non-NF1 patients revealed some EGF-R expression (46). Our analysis of Schwann cells isolated from NF1-mutant mouse embryos demonstrated that one of the events occurring concomitant with transformation of $(-/-)$ cells is expression of the EGF-R. The TXF cells, which do not arise from wild-type $(+/+)$ embryos, remained $\mathrm{S}_{100}+\mathrm{GGF}$ responsive and expressed other Schwann cell markers (23). Thus both the human and mouse systems indicate that mutational loss of NF1 may lead to the development of altered Schwann cells with EGF-R expression. It is difficult at present to assess the role of the EGF- $\mathrm{R}^{+} / \mathrm{S}_{100}{ }^{+}$cells in the development and progression of benign neurofibromas. Although these cells make up only a small portion of the total population, they may be altered in their interactions with other cells in the tumor environment or may release factors that help drive the growth of benign tumors, which typically undergo periodic bursts of growth. Alternatively, the EGF- $\mathrm{R}^{+}$cells may only arise after the benign tumor is formed. Similarly, it is unknown what potential ligands for the EGF-R may be present in benign neurofibromas, although they do contain a rich diversity of cell types, one or more of which may produce an EGF-R ligand. Whereas the vast majority of neurofibromas do not progress to malignancy, they represent the primary burden for many patients, and the role of the EGF- $\mathrm{R}^{+} / \mathrm{S}_{100}{ }^{+}$cells in these tumors demands further investigation.

These studies also raise questions about the role of EGF-R in tumor progression, specifically whether EGF$\mathrm{R}$ expression is a necessary step in MPNST formation. All three NF1 patient's MPNST lines expressed EGF-R, and seven of seven primary NF1 MPNST analyzed by immunohistochemistry contained EGF-R ${ }^{+}$cells. However, the relative levels of EGF-R expression in individual cells varied, as did the proportion of cells expressing EGF-R in each tumor. The existence of microdomains defined by EGF-R expression suggests heterogeneity within individual MPNSTs. It is possible that EGF-R expression is transient and occurs at particular stages in tumorigenesis or that expression of the EGF-R represents only one of several routes to formation of MPNST. The significance of EGF-R expression in Schwann cell tumorigenesis is strengthened by our finding that both the $\mathrm{S}_{100}$, Schwann cell-derived rat $\mathrm{RN}-22$ line and the $\mathrm{S}_{100^{+}}$, non-NF1 human MPNST line S-26T express EGF-R. The finding of EGF-R expression in such a diversity of settings strongly implicates a role for it in the pathogenesis of malignant nerve sheath tumors.

One important distinction between the mouse and human systems was that the three NF1 MPNST lines were $\mathrm{S}_{100}$, and two of three lacked erbB3 expression and GGF-responsiveness. If these results are interpreted in terms of the Schwann cell origin model, they suggest that subsequent to acquisition of EGF-R expression, the cells begin to dedifferentiate and lose markers such as erbB3 and S100. Such a model may also help explain the general (though not complete) discordance between S100 and EGF-R expression among cells in primary MPNST samples and the fact that 5 of the 7 samples lacked S100 expression. Interestingly, both $\mathrm{RN}-22$ and S-26T were unresponsive to GGF, suggesting that loss of erbB3 may contribute to the dedifferentiation of cells during tumorigenesis even without loss of S100. Our finding that NF1-deficient mouse Schwann cells, human $\mathrm{S}{ }^{+} 0^{+}$Schwann cells from neurofibromas, and RN-22 cells all express EGF-R leads us to favor the hypothesis that loss of Schwann cell markers is a feature of MPNST formation that can occur subsequent to expression of EGF-R. An alternative model would suggest that the cells giving rise to the $\mathrm{S} 100^{+} /$EGF- $^{+}$cells in benign neurofibromas and/or to the cells in MPNST, represent a different lineage arising from the neural crest. Such an argument could also be applied to the mouse TXF (-/-) cells. In each case, the loss of NF1 would lead to a sub-population of cells that acquire EGF-R and various combinations of Schwann cell markers that might change during the evolution of the tumor. This alternative model is being evaluated in our current studies employing microchip gene arrays to compare and contrast the overall pattern of genes expressed in (+/+), (-/-), and TXF (-/-) cells. Another area of future investigation will be to determine the degree to which the EGF-R exerts its mitogenic effects through the Ras pathway. It is interesting that the expression of EGF-R occurs in the context of cells that already have high levels of Ras-GTP because of loss of NF1, and this may suggest that the signaling from the EGF-R also involves non-Ras pathways, thereby augmenting the high Ras activity present in the cells. 


\section{Acknowledgments}

We thank Doug Lowy, Isabel Martinez-Lacaci, and David Salomon for comments and suggestions; Ricardo Dreyfuss for excellent assistance with photography; and Mary Ann Miller for expert assistance with immunohistochemistry. We are grateful to Bruce Korf and Gretchen M. Schneider (Boston Children's Hospital, Boston, Massachusetts, USA) and the University of Miami Human Tissue Bank (Miami, Florida, USA) for providing neurofibromas. We thank Patrick Wood (The Miami Project, Miami, Florida, USA) for normal human nerves, and Diya Mutasim (University of Cincinnati, Department of Dermatology, Cincinnati, Ohio, USA) and Kevin Bove (Cincinnati Children's Hospital, Department of Pathology, Cincinnati, Ohio, USA) for neurofibroma sections. The University of Alabama (Birmingham, Alabama, USA) and the Childhood Cancer Tissue Network (Columbus, Ohio, USA) provided MPNST. N. Ratner is supported by National Institutes of Health grant NS-28840.

1. Huson, S.M., Harper, P.S., and Compston, D. 1988. Von Recklinghausen neurofibromatosis: a clinical and population study in south-east Wales. Brain. 111:1355-1381.

2. Upadhyaya, M., and Cooper, D.N. 1998. Neurofibromatosis type 1: from genotype to phenotype. BIOS Scientific Publishers Ltd. Oxford, United Kingdom. pp. 230.

3. Ferner, R.E. 1998. Clinical aspects of neurofibromatosis 1. In Neurofibromatosis type 1: from genotype to phenotype. M. Upadhyaya and D.N. Cooper editors. BIOS Scientific Publishers Ltd., Oxford, United Kingdom. 21-38.

4. Rosenbaum, T., Petrie, K.M., and Ratner, N. 1997. Neurofibromatosis type 1: genetic and cellular mechanisms of peripheral nerve tumor formation. The Neuroscientist. 3:412-420.

5. Stefansson, K., Wollmann, R., and Jerkovic, M. 1982. S-100 protein in soft tissue tumours derived from Schwann cells and melanocytes. Am.J. Pathol. 106:261-268.

6. Peltonen, J., et al. 1988. Cellular differentiation and expression of matrix genes in type 1 neurofibromatosis. Lab. Invest. 59:760-761.

7. Sheela, S., Riccardi, V.M., and Ratner, N. 1990. Angiogenic and invasive properties of neurofibroma Schwann cells. J. Cell Biol. 111:645-653.

8. Ducatman, B.S., Scheithauer, B.W., Piepgras, D.G., and Reiman, H.M 1984. Malignant peripheral nerve sheath tumors in childhood. J. Neurooncol. 2:241-248.

9. Zoller, M.E., Rembeck, B., Oden, A., Samuelsson, M., and Angervall, L. 1997. Malignant and benign tumors in patients with neurofibromatosis type 1 in a defined Swedish population. Cancer. 79:2125-2131.

10. Side, L.E., and Shannon, K.M. 1998. The NF1 gene as a tumor suppressor. In Neurofibromatosis type 1: from genotype to phenotype. M. Upadhyaya and D.N. Cooper, editors. BIOS Scientific Publishers Ltd. Oxford, United Kingdom. 133-152.

11. Morioka, N., Tsuchida, T., Etoh, T., Ishibashi, Y., and Otsuka, F. 1990. A case of neurofibrosarcoma associated with neurofibromatosis: light microscopic, ultrastructural, immunohistochemical and biochemical investigations. J. Dermatol. 17:312-316.

12. Wallace, M.R., et al. 1990. Type 1 neurofibromatosis gene: identification of a large transcript disrupted in three NF1 patients. Science. 249:181-186

13. Cawthon, R., et al. 1990. A major segment of the neurofibromatosis type 1 gene; cDNA sequence, genomic structure, and point mutations. Cell. 62:193-201.

14. Viskochil, D., et al. 1990. Deletions and a translocation interrupt a cloned gene at the neurofibromatosis type 1 locus. Cell. 62:187-192.

15. Upadhyaya, M., and Cooper, D.N. 1998. The mutational spectrum in neurofibromatosis 1 and its underlying mechanisms. In Neurofibromatosis type 1: from genotype to phenotype. M. Upadhyaya and D.N. Cooper, editors. BIOS Scientific Publishers Ltd. Oxford, United Kingdom. 65-88.

16. Boguski, M.S., and McCormick, F. 1993. Proteins regulating Ras and its relatives. Nature. 366:643-654

17. DeClue, J.E., et al. 1992. Abnormal regulation of mammalian $\mathrm{p} 21^{\text {ras }}$ contributes to malignant tumor growth in von Recklinghausen (type-1) neurofibromatosis. Cell. 69:265-273.
18. Basu, T.N., et al. 1992. Aberrant regulation of ras proteins in malignant umour cells from type-1 neurofibromatosis patients. Nature. 356:713-715

19. Guha, A., et al. 1996. Ras-GTP levels are elevated in human NF1 peripheral nerve tumors. Oncogene. 12:507-513.

20. Jacks, T., et al. 1994. Tumor predisposition in mice heterozygous for a targeted mutation in NF1. Nat. Genet. 7:353-361.

21. Brannan, C.I., et al. 1994. Targeted disruption of the neurofibromatosis type- 1 gene leads to developmental abnormalities in heart and various neural crest-derived tissues. Genes Dev. 8:1019-1029.

22. Kim, H., et al. 1995. Schwann cells from neurofibromin deficient mice exhibit activation of $\mathrm{p}^{2} 1^{\text {ras }}$, inhibition of cell proliferation and morphological changes. Oncogene. 11:325-335.

23. Kim, H.A., Ling, B., and Ratner, N. 1997. NF1-deficient mouse Schwann cells are angiogenic and invasive and can be induced to hyperproliferate: reversion of some phenotypes by an inhibitor of farnesyl transferase. Mol. Cell. Biol. 177:862-872.

24. Levi, A.D., et al. 1995. The influence of heregulins on human Schwann cell proliferation. J. Neurosci. 15:1329-1340.

25. Pinkas-Kramarski, R., Alroy, I., and Yarden, Y. 1997. ErbB receptors and EGF-like ligands: cell lineage determination and oncogenesis through combinatorial signaling. J. Mammary Gland Biol. Neoplasia. 2:97-107.

26. Burden, S., and Yarden, Y. 1997. Neuregulins and their receptors: a versatile signaling molecule in organogenesis and oncogenesis. Neuron. 18:847-855.

27. Kim, H., DeClue, J.E., and Ratner, N. 1997. cAMP-dependent protein kinase A is required for Schwann cell growth: interaction between cAMP and neuregulin/tyrosine kinase pathways. J. Neurosci. Res. 49:236-247.

28. Colman, S.D., Williams, C.A., and Wallace, M.R. 1995. Benign neurofibromas in type 1 neurofibromatosis (NF1) show somatic deletions of the NF1 gene. Nat. Genet. 11:90-92.

29. Sawada, S., et al. 1996. Identification of NF1 mutations in both alleles of a dermal neurofibroma. Nat. Genet. 14:110-112.

30. Serra, P., et al. 1997. Confirmation of a double-hit model for the NF1 gene in benign neurofibromas. Am. J. Hum. Genet. 61:512-519.

31. Menon, A.G., et al. 1990. Chromosome 17p deletions and p53 gene mutations associated with the formation of malignant neurofibrosarcomas in von Recklinghausen neurofibromatosis. Proc. Natl. Acad. Sci. USA. 87:5435-5439.

32. Velu, T.J., et al. 1989. Retroviruses expressing different levels of the normal epidermal growth factor receptor: biological properties and new bioassay. J. Cell. Biochem. 39:153-166.

33. Gutmann, D.H., et al. 1997. The diagnostic evaluation and multidisciplinary management of neurofibromatosis 1 and neurofibromatosis 2 . JAMA. 278:51-57.

34. Dahlberg, W.K., Little, J.B., Fletcher, J.A., Suit, H.D., and Okunieff, P. 1993. Radiosensitivity in vitro of human soft tissue sarcoma cell lines and skin fibroblasts derived from the same patients. Int. J. Radiat. Biol. 63:191-198.

35. Merlino, G.T., et al. 1985. Structure and localization of genes encoding aberrant and normal epidermal growth factor receptor RNAs from A431 human carcinoma cells. Mol. Cell. Biol. 5:1722-1734.

36. Merlino, G.T., et al. 1984. Amplification and enhanced expression of the epidermal growth factor receptor gene in A431 human carcinoma cells. Science. 224:417-419.

37. Pfeiffer, S.E., and Wechsler, W. 1972. Biochemically differentiated neoplastic clone of Schwann cells. Proc. Natl. Acad. Sci. USA. 69:2885-2889.

38. Peng, D., et al. 1996. Anti-epidermal growth factor receptor monoclonal antibody 225 up-regulates $\mathrm{p}^{27 \mathrm{KIP} 1}$ and induces $\mathrm{G}_{1}$ arrest in prostatic cancer cell line DU145 ${ }^{1}$. Cancer Res. 56:3666-3669.

39. Levitzki, A., and Gazit, A. 1995. Tyrosine kinase inhibition: an approach to drug development. Science. 267:1782-1788.

40. Garcia, A.M., Rowell, C., Ackermann, K., Kowalczyk, J.J., and Lewis, M.D. 1993. Peptidomimetic inhibitors of Ras farnesylation and function in whole cells. J. Biol. Chem. 268:18415-18418.

41. Yan, N., et al. 1995. Farnesyltransferase inhibitors block the neurofibromatosis type I (NF1) malignant phenotype. Cancer Res. 55:3569-3575.

42. Salomon, D.S., Brandt, R., Ciardiello, F., and Normanno, N. 1995. Epidermal growth factor-related peptides and their receptors in human malignancies. Crit. Rev. Oncol. Hematol. 19:183-232.

43. Bridges, A.J. 1996. The epidermal growth factor receptor family of tyrosine kinases and cancer: can an atypical exemplar be a sound therapeutic target? Curr. Med. Chem. 3:167-194.

44. Cichowski, K., et al. 1999. Mouse models of tumor development in neurofibromatosis type 1. Science. 286:2172-2176.

45. Vogel, K.S., et al. 1999. Mouse model for neurofibromatosis type 1. Science. 286:2176-2179.

46. Sturgis, E.M., Woll, S.S., Aydin, F., Marroji, A.J., and Amedee, R.G. 1996. Epidermal growth factor receptor expression by acoustic neuromas. Laryngoscope. 106:457-462. 